\title{
Anomalous Electron Trajectory in Topological Insulators
}

\author{
Likun Shi ${ }^{1}$, Shoucheng Zhang ${ }^{2}$, Kai Chang ${ }^{1}$ \\ ${ }^{1}$ SKLSM, Institute of Semiconductors, Chinese Academy of Sciences, P.O. Box 912, Beijing 100083, China and \\ ${ }^{2}$ Department of Physics, Stanford University, Stanford, CA94305
}

\begin{abstract}
We present a general theory about electron orbital motions in topological insulators. An in-plane electric field drives spin-up and spin-down electrons bending to opposite directions, the skipping orbital motions, a counterpart of the integer quantum Hall effect, are formed near the boundary of the sample. The accompanying Zitterbewegung can be found and controlled by tuning external electric fields. Ultrafast flipping electron spin leads to a quantum side jump in topological insulator, and a snake orbit motion in two-dimensional electron gas with spin-orbit interactions. This feature provides us a new way to control electron orbital motion by manipulating electron spin.
\end{abstract}

PACS numbers: 71.70.Ej, 75.76.+j, 72.25.Mk

The time-reversal invariant topological insulator (TI) is a new state of quantum matter possessing insulating bulk and metallic edge or surface states, which shows a linear massless Dirac dispersion [1, 2]. TIs are distinguished from a normal band insulator by a nontrivial topological invariant $Z_{2}$ characterizing its band structure. The quantum spin Hall effect was proposed in graphene [3] and HgTe quantum wells [4] . The existence of edge and surface states was confirmed by the recent experiment in $\mathrm{HgTe}$ quantum wells [5] and the angle-resolved photoemission spectroscopy experiments $[6$, 7]. Due to its unique band structure, TI is a good testbed for observing relativistic effects, predicted by the Dirac equation. For instance, the Klein's paradox and Zitterbewegung (ZB).

So far, the most previous works in the rapid growing field of TI focused on exploring new TIs and its transport and magnetic properties. Relatively, electron dynamics in TIs is unexplored. In this work we show that the quantum spin Hall effect and quantum anomalous Hall effect [8] can be understood from anomalous electron orbital motions in TIs. These anomalous electron orbital motions in topological insulators give naturally a clear picture about the origin of the edge states, a counterpart of the skipping orbital motion in the integer quantum Hall effect. By applying a series of magnetic field pulses to flip electron spin quickly, a quantum side-jump behavior and a snake-orbit motion can be found for electrons in TIs and normal 2DEG with the spin-orbit interactions (SOIs), respectively. The trembling motion, i.e., the ZB, can be controlled by changing an in-plane electric field and the initial momentum of the electron wavepacket.

We consider the single-particle Hamiltonian of electron at low-energy regime in the presence of a uniform electric field $\boldsymbol{E}$

$$
\begin{aligned}
H & =H_{0}(k)+V(\boldsymbol{r}), \\
& =\epsilon(k) I+\sum_{i=1}^{3} d_{i}^{0}(k) \sigma_{i}-e \boldsymbol{E} \cdot \boldsymbol{r},
\end{aligned}
$$

where $\sigma_{i}(i=1,2,3)$ is the Pauli matrix, $\epsilon(k)$ is the ki- netic energy. The different forms of the $d_{i}$ can be used to describe the various important systems: 1) the twodimensional electron gas with the Rashba and Dresselhaus SOIs with $d_{1}^{0}=-\alpha k_{y}-\beta k_{x}, d_{2}^{0}=\alpha k_{x}+\beta k_{y}$, $d_{3}^{0}=0$, where $\alpha$ and $\beta$ describe the strengths of the Rashba and Dresselhaus SOIs, respectively [9]; 2) single layer graphene with $d_{1}^{0}=v_{F} \hbar k_{x}, d_{2}^{0}=v_{F} \hbar k_{y}, d_{3}^{0}=0$ [10]; 3) bilayer graphene with $d_{1}^{0}=-\hbar^{2}\left(k_{x}^{2}-k_{y}^{2}\right) / 2 m$, $d_{2}^{0}=-\hbar^{2} k_{x} k_{y} / m, d_{3}^{0}=0$ [10]; 4) the two-dimensional TI HgTe quantum wells with an inverted band structure with $d_{1}^{0}=A k_{x}, d_{2}^{0}=A k_{y}, d_{3}^{0}=M-B k^{2}$ [4] ; 5) threedimensional TIs, e.g., $\mathrm{Bi}_{2} \mathrm{Se}_{3}$ and $\mathrm{Bi}_{2} \mathrm{Te}_{3}$, whose Hamiltonian would be extended to $H=\sum_{\mu, \nu=0}^{3} d_{\mu \nu}^{0}(k) \sigma_{\mu} \otimes \sigma_{\nu}-$ $e \boldsymbol{E} \cdot \boldsymbol{r}$, in which $\sigma_{0}=I_{2 \times 2}$, and $d_{00}^{0}=\epsilon(k), d_{03}^{0}=M(k)$, $d_{31}^{0}=A_{1} k_{z}, d_{11}^{0}=A_{2} k_{x}, d_{12}^{0}=A_{2} k_{y}$ while other $d_{\mu \nu}^{0} \mathrm{~s}$ are zero [11, 12].

In the absence of a uniform electric field, the electron position operator $y_{H}(t)$ evolving with the time $t$ can be obtained as

$$
\begin{aligned}
y_{H}(t) & =e^{i H t / \hbar} y e^{-i H t / \hbar}, \\
& =y(0)+(i t / \hbar)[H, y]+\frac{(i t / \hbar)^{2}}{2 !}[H,[H, y]]+\cdots, \\
& =y(0)+(i t / \hbar) \epsilon^{y}+\sum_{n=1} \frac{(i t / \hbar)^{n}}{n !} T_{n},
\end{aligned}
$$

where $\epsilon^{y}=[\epsilon, y]=-i \partial_{k_{y}} \epsilon, \quad D^{0}=\sum_{j} d_{j}^{0} \sigma_{j}$, $T_{1}=\left[D^{0}, y\right]=\sum_{j} d_{j}^{y} \sigma_{j}=D^{y}, T_{2}=\left[D^{0}, D^{y}\right]=$ $\sum_{i, j} d_{i}^{0} d_{j}^{y}\left[\sigma_{i}, \sigma_{j}\right], \cdots$, the $n$-th commutator $T_{n}=$ $\left[D^{0}, T_{n-1}\right]$. Generally, the analytical expression of the above summation is very difficult to obtain because the number of the commutators increases hierarchically with increasing the order $n$.

Interestingly, the equivalence between the commutation relationship $\left[\sigma_{i}, \sigma_{j}\right]=2 i \varepsilon_{i j k} \sigma_{k}$ and the vector cross production $(\boldsymbol{A} \times \boldsymbol{B})_{k}=\varepsilon_{i j k} A_{i} B_{j} \mathbf{e}_{k}$ (see the online supplementary material) offers us a new way to solve this problem. In the absence of an external electric field, the commutation between the spin operators is converted into the vector product between them. We can transfer 


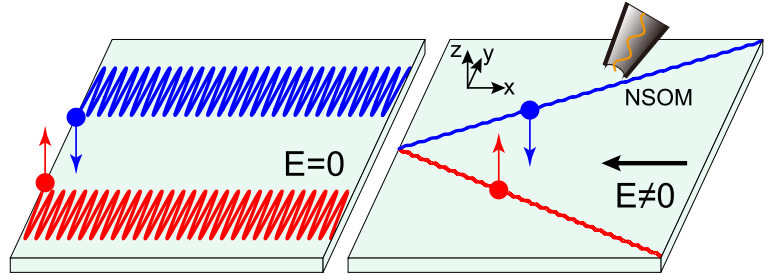

(a)

(b)

FIG. 1: (Color online) Schematic of electron orbital motion in a HgTe quantum well with an inverted band without and with an in-plane driving electric field [(a) and (b)]. The orbital motion can be detected by the optical technique, e.g., the NSOM at the microwave frequency regime.

the algebra summation in Eq. (2) into the summations of the vector series $T_{2 n+1}\left(T_{2 n}\right)(n=1,2, \cdots)$ utilizing the equivalence between the commutator $\left[D^{0}, T_{n}\right]$ and the vector product $D^{0} \times T_{n}, T_{n}=\left[D_{0}, T_{n-1}\right] \sim 2 i\left(D^{0} \times T_{n-1}\right)$ (see the online supplementary material). One can see that the vectors $T_{2 n}$ and $T_{2 n+1}(n=0,1,2, \ldots)$ point along the orthogonal directions while the lengths of the vectors $T_{n}$ varies with the increasing of the order $n$ as $T_{2 n+1}=-\left|2 D^{0}\right|^{2} T_{2 n-1}$, where $\left|D_{0}\right|=\left(\sum_{i=1}^{3}\left(d_{i}^{0}\right)^{2}\right)^{1 / 2}$. This character allows us to get the analytical expression for the electron position operator

$$
\begin{aligned}
y_{H}(t) & =y(0)+\frac{i t}{\hbar}\left(\epsilon^{y}+T_{1}-\frac{T_{3}}{\left|D^{0}\right|^{2}}\right) \\
& +\frac{T_{2}}{2\left|D^{0}\right|^{2}}\left[\cos \left(2\left|D^{0}\right| t / \hbar\right)-1\right] \\
& +\frac{i T_{3}}{2\left|D^{0}\right|^{3}}\left[\sin \left(2\left|D^{0}\right| t / \hbar\right)\right] .
\end{aligned}
$$

This analytical expression consists of the initial position (the first term), the propagating term (the second term) and the Zitterbewegung term (the last two terms). This ZB term describes the rapid trembling motion which has no the classical correspondences. ZB, a novel relativistic quantum orbital motion, is an inherent hallmark of the Dirac equation, first predicted by Schrödinger in 1930 [13]. This rapid quivering motion of electron arises from the superposition between the positive- and negative- energy part of the spinor states and could be a possible origin of electron spin [14]. Although there are many proposals about observation of the $\mathrm{ZB}$ in a two-dimensional electron gas (2DEG) with the SOIs 9, 15], graphene [10] and a Cooper pair in superconductors [16, 17] and related theoretical analysis [18], but only observed experimentally in the trapped ion [19 21] and cold atom 22] systems. However, this prediction has still never been observed for electrons in free space, this is because the $\mathrm{ZB}$ displays an extremely high frequency $\omega_{Z}=2 m_{0} c^{2} / \hbar \approx 1.5 \times 10^{21} \mathrm{~Hz}$ and a tiny amplitude $\lambda_{Z}=\hbar / m_{0} c \approx 3.9 \times 10^{-4} \mathrm{~nm}\left(m_{0}\right.$ is the electron mass $)$. The detection of the oscillation with such high frequency and negligibly small amplitude is beyond the reach of the present-day experimental technique.

It is natural to imagine that the frequency of the ZB should be decreased when the energy gap decreases. TI could be a good testbed to observe the $\mathrm{ZB}$ due to its narrow bulk gap which ranges from $10 \mathrm{meV}$ to $0.3 \mathrm{eV}$. The expression of ZB (see Eq. [3]) agrees exactly with the previous theoretical works for various systems. For a 2DEG with the Rashba and Dresselhaus SOIs [9], $\epsilon^{y}+$ $T_{1}=-i \hbar^{2} k_{x} / m-i\left(\alpha \sigma_{y}-\beta \sigma_{x}\right), T_{2}=\left(\alpha^{2}-\beta^{2}\right) k_{y} \sigma_{z}$, $T_{3}=\left(\alpha^{2}-\beta^{2}\right)\left(k_{x} \sigma_{y}-k_{y} \sigma_{x}\right) \Sigma$, where $\Sigma=\alpha\left(k_{x} \sigma_{x}+\right.$ $\left.k_{y} \sigma_{y}\right)+\beta\left(k_{x} \sigma_{y}+k_{y} \sigma_{x}\right),\left|D^{0}\right|=\left(\alpha^{2}+\beta^{2}\right) k^{2}+4 \alpha \beta k_{x} k_{y}$. For a single layer graphene [10], $\epsilon^{y}+T_{1}=-i \hbar v_{F} \sigma_{x}, T_{2}=$ $\hbar^{2} v_{F}^{2} k_{y} \sigma_{z}, T_{3}=\hbar^{3} v_{F}{ }^{3} k_{y}\left(k_{x} \sigma_{y}-k_{y} \sigma_{x}\right),\left|D^{0}\right|=v_{F} \hbar k$. The two-dimensional TI HgTe quantum wells with an inverted band structure [4], $\epsilon^{y}+T_{1}=-i\left(2 D k_{y}+A \sigma_{y}\right)$, $T_{2}=-2 A\left(M-B k^{2}\right) \sigma_{x}+2 A^{2} k_{x} \sigma_{z}, T_{3}=4 i\left\{A^{3} k_{x} k_{y} \sigma_{x}-\right.$ $\left.\left[A\left(M-B k^{2}\right)^{2}+A^{3} k_{x}^{2}\right] \sigma_{y}+A^{2}\left(M-B k^{2}\right) k_{y} \sigma_{z}\right\}$ (see Fig. 1(a)). The second term $\epsilon^{y}+T_{1}-T_{3} /\left|D^{0}\right|^{2}$ represents the classical uniform rectilinear motion, the third and the fourth terms $T_{2}$ and $T_{3}$ describe the $\mathrm{ZB}$ with the oscillating frequency $2\left|D^{0}\right| / \hbar$. The trembling frequency is determined by the strength of the spin-orbit interaction of the interband coupling $d_{i}^{0}$. For all above examples, these agreements demonstrate the validity of the diagram technique developed by us.

In two-terminal transport experiments, an external voltage is applied between the source and drain, generating an in-plane electric field. In the presence of a uniform in-plane electric field, it is difficult to get the analytical expression of the electron position operator as discussed above. Instead, we can calculate the electron position $\mathbf{r}(t)$ based on the equation of motion $i \hbar \dot{\mathbf{r}}=[\mathbf{r}, H]$. We consider a two-dimensional topological insulator, a $\mathrm{HgTe}$ quantum well with an inverted band structure described by the BHZ model [4]. The single-particle effective Hamiltonian for the electron is

$$
H(k)_{\uparrow \downarrow}=H_{0}(k)_{\uparrow \downarrow}+V\left(x_{i}\right),
$$

where $H_{0}(k)_{\uparrow \downarrow}=C-D k^{2} \pm A k_{x} \sigma^{x}+A k_{y} \sigma^{y}+(M-$ $\left.B k^{2}\right) \sigma^{z}, V(\mathbf{x})=-e \mathbf{E} \cdot \mathbf{r} \sigma^{z} . A, B, C, D$, and $M$ are the parameters determined by the thickness of the quantum well 23]. Consider an electron injected in the Gaussian wave packet $\varphi(r)=\frac{1}{2 \pi} \frac{d}{\sqrt{\pi}} \int d^{2} k e^{-d^{2}\left(\vec{k}-\vec{k}_{0}\right)^{2} / 2} e^{i \vec{k} \vec{r}}|\uparrow\rangle$ with the spatial width $(\Delta x)^{2}=(\Delta y)^{2}=d^{2} / 2$ and spin pointing along the $z$ axis, perpendicular to the quantum well plane. The guiding center of the wave packet $\left\langle y_{H}(t)\right\rangle$ can be calculated numerically by the Heisenberg equation of motion $d\left\langle y_{H}(t)\right\rangle / d t=(i h)^{-1}\left\langle\left[y_{H}(t), H\right]\right\rangle$. From the numerical results (the red and blue curves in Fig. 1(c)), the spin-up and spin-down electron driven by an in-plane electric field $E_{x}$ bend to the opposite direction along the $y$ axis accompanying with a trembling behavior, i. e., the ZB. Notice that the electron energy $\langle E\rangle=C+M-$ $(B+D) / d^{2}-(B+D) k_{0}^{2}$ locates in the bulk gap, which 
is the important difference from the spin Hall effect in a conventional 2DEG and/or metal with the SOIs.

In order to understand this surprising feature, we analyze the equation of motion utilizing the adiabatic approximation, i.e., separating the fast trembling motion and slow orbital motion $y_{\uparrow \downarrow}=y_{\uparrow \downarrow}^{\mathrm{orb}}+y_{\uparrow \downarrow}^{\mathrm{ZB}}[24,25]$. First we perform a unitary transformation $U(k)$ to diagonalize the Hamiltonian $H_{0}(k)$, i. e., $\tilde{H}_{0}=U(k)_{\uparrow \downarrow} H_{0}(k)_{\uparrow \downarrow} U^{\dagger}(k)_{\uparrow \downarrow}$. The potential term becomes $V\left(\tilde{D}_{i}\right)$, where the covariant derivative $\tilde{D}_{i}=i \partial_{k_{i}}-\tilde{A}_{i}$, and $\tilde{A}_{i}(k)_{\uparrow \downarrow}=-i$. $U(k)_{\uparrow \downarrow} \partial_{k_{i}} U^{\dagger}(k)_{\uparrow \downarrow} \cdot U(k)_{\uparrow \downarrow} \sigma^{z} U^{\dagger}(k)_{\uparrow \downarrow}$ behaves like a gauge field. Adopting the adiabatic approximation, i.e., neglecting the off-diagonal matrix elements of $\tilde{A}_{i}$, the resulting gauge field $A_{i}$ only contains a diagonal matrix which gives a non-zero associated field strength $F_{i j}=$ $i\left[D_{i}, D_{j}\right]$, where $x_{i} \rightarrow D_{i}=i \partial_{k_{i}}-A_{i}$, then the effective Hamiltonian becomes

$$
H_{\uparrow \downarrow}^{\mathrm{eff}}=\tilde{H}_{0}(k)-e \sum_{i=x, y} E_{i} D_{i, \uparrow \downarrow}
$$

where $\tilde{H}_{0}(k)=C-D k^{2}-\sqrt{A^{2} k^{2}+\left(M-B k^{2}\right)^{2}} \sigma^{z}$ for both spin-up (down) states. The nontrivial property of the Hamiltonian is revealed through the nontrivial commutation relations $\left[k_{i}, k_{j}\right]=0,\left[D_{i}, k_{j}\right]=i \delta_{i j}$, $\left[D_{i}, D_{j}\right]=-i F_{i j}$, the effective "Lorentz forces" in momentum space felted by spin-up or spin-down electron is

$$
F_{x y}(k)_{\uparrow \downarrow}= \pm \frac{A^{2}\left(M^{2}-B^{2} k^{4}\right)}{2\left[A^{2} k^{2}+\left(M-B k^{2}\right)^{2}\right]^{2}} .
$$

The equation of motion for the spin-up/down electron can be written as

$$
\begin{aligned}
\dot{x}_{\uparrow \downarrow} & =u_{x} \pm\left|F_{x y}\right| \dot{k}_{y}, \\
\dot{y}_{\uparrow \downarrow} & =u_{y} \mp\left|F_{x y}\right| \dot{k}_{x}, \\
k_{i} & =k_{i 0}+\lambda e E_{i} t / \hbar,
\end{aligned}
$$

where $u_{i}$ represents the usual group velocity $\partial \tilde{H}_{0} / \partial k_{i}$, and $\lambda= \pm 1$ stands for the negative (positive) branch of the energy spectrum. When $k_{y 0}=0$, the orbital trajectory of spin-up or spin-down electron at small $k$ is (see the online supplementary material)

$$
y_{\uparrow \downarrow}^{\text {orb }}=\mp \frac{A^{2}}{2 M^{2}} k_{x}+O(k)^{3} .
$$

Clearly, one can see that the spin-up and spin-down electrons feel an opposite force $F_{x y}(k)_{\uparrow \downarrow}$, respectively, which pushes them against the opposite direction. The trajectories obtained from Eq. [7], which neglects the fast trembling motion by the adiabatic approximation. The analytical expression for the trembling motion, i.e., the ZB, at small $k$ can also be obtained (see the online supplementary material)

$$
y_{\uparrow \downarrow}^{\mathrm{ZB}}= \pm \frac{A^{2}}{2 M^{2}} \frac{e E_{x}}{\Delta \epsilon(t)} \sin \left(\frac{\Delta \epsilon(t)}{\hbar} t\right),
$$

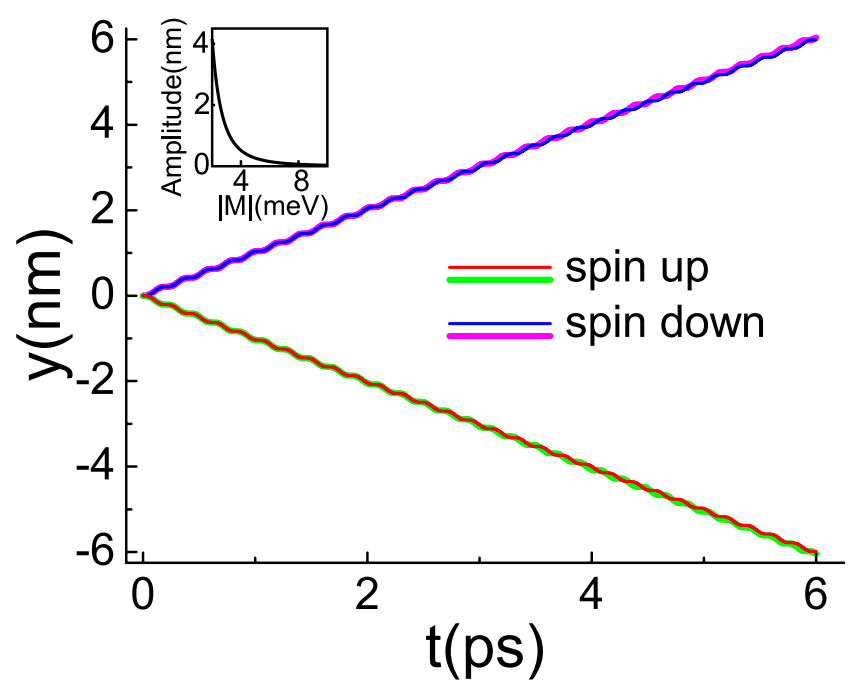

FIG. 2: (color online). The trajectories of spin-up (the red and green curves) and spin-down (the blue and purple curves) electrons incident with the initial momenta $k_{x 0}=0.001 \mathrm{~nm}^{-1}$, the electric field $E_{x}=10 \mathrm{~V} / \mathrm{cm}$. The red and blue (green and purple) curves denote the numerical (analytical) results. The parameters used in the calculation are adopted from [23]. The inset shows that the amplitude of the transverse $\mathrm{ZB} y^{\mathrm{ZB}}$ as a function of the bulk gap of $\mathrm{HgTe} \mathrm{QW}|M|$.

where $\Delta \epsilon(t)=2 \sqrt{A^{2} k^{2}+\left(M-B k^{2}\right)^{2}}=2|M|+O\left(k^{2}\right)$. At small in-plane momentum $k$, the kinetic energy can be neglected, the frequency of amplitude of the ZB are both determined by $M$, the gap of $\mathrm{HgTe} \mathrm{QW}$ which can be tuned by the thickness of the QW. The total electron trajectories $y_{\uparrow \downarrow}=y_{\uparrow \downarrow}^{\mathrm{orb}}+y_{\uparrow \downarrow}^{\mathrm{ZB}}$ obtained from Eq. [7] and [8] agree well with the numerical results for spin-up and spin-down electron incident cases (see Fig. 1(c)). The inset in Fig. 1(c) shows the amplitude of the ZB increases rapidly with decreasing the gap $|M|$ of $\mathrm{HgTe} \mathrm{QW}$ with an inverted band, and a linear dependence on the strength of the in-plane electric field $E_{x}$. For example, the amplitude of the ZB is about $21.2 \AA$ when $M=2.5 \mathrm{meV}$ and $E_{x}=$ $10 \mathrm{~V} \mathrm{~cm}^{-1}$, which are within the reach of the state-of-art experimental techniques. By adjusting the electric fields $E_{x}$ and the bulk gap of $\mathrm{HgTe} \mathrm{QWs}$, one can tune the amplitude of the ZB significantly (see Fig. 2(a)), and make it possible to observe it in TIs.

For the realistic experimental sample, we need to consider the boundary effect, for instance, a spin Hall bar with a finite width. The spin-up and spin-down electrons will bend to opposite edges. Fig. 3 (b) shows clearly that spin-up and spin-down edge states appear near the opposite boundaries of the sample (the shaded regions). From the inset, one can see the skipping orbital motion of electrons in the edge states, since electrons bending to the edges will be bounced back when it hits the hard-wall boundary [26], and pushed to the boundary again by the driving force (or the effective "Lorentz" force) $F_{x y}(k)_{\uparrow \downarrow}$. This is a counterpart of the skipping 


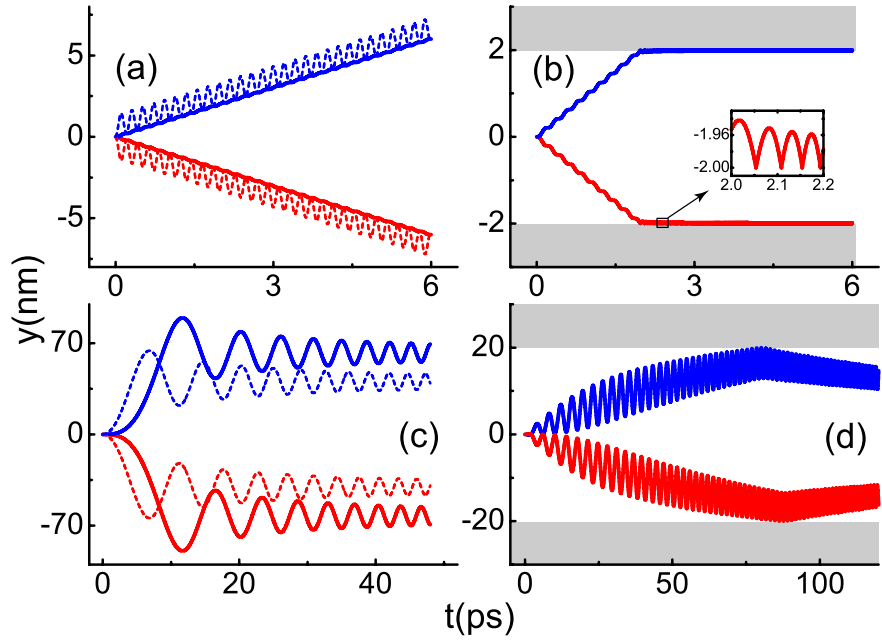

FIG. 3: (color online). The trajectories of spin-up (red) and spin-down (blue) incident electrons under a uniform electric field. The in-plane electric field $E=10 \mathrm{~V} \mathrm{~cm}{ }^{-1}$. The electron injected into $\mathrm{HgTe}$ quantum well $(M=-10 \mathrm{meV})$ (a) has a initial incident momentum $k_{x 0}=0$ for the solid curves and $k_{x 0}=0.001 \mathrm{~nm}^{-1}$ for the dashed curves; (b) The same as (a), but with a hard-wall boundary (the shaded regions). The inset amplifies the trajectory near the boundary, showing the skipping orbital motion. (c) and (d) The same as (a) and (b), but in a conventional GaAs 2DEG with the Rashba spinorbit interaction $(\alpha=10 \mathrm{meV} \mathrm{nm}$ taken from Ref. [4]). The solid and dashed curves corresponds to $k_{x 0}=0$ and $k_{x 0}=$ $0.01 \mathrm{~nm}^{-1}$, respectively.

orbital motion in the integer quantum Hall effect, but spin-up and spin-down electrons feel opposite the effective "Lorentz" forces. While in a normal 2DEG with the SOIs, spin-up and spin-down electrons show a ballistic side jump in opposite directions which was already found before [15], but no edge states can be found near the sample boundary. Instead, electrons will oscillate between the opposite boundaries (see Figs. 3(c) and 3(d)).

Similarly, the quantum anomalous spin Hall effect might also be understood from the electron dynamics in TIs. Considering a ferromagnetic TI, a giant Zeeman splitting results in a spin-up inverted and a spin-down normal band structure, we assume these magnetic ions polarized along $z$ axis. This situation corresponds to the BHZ Hamiltonian with the positive and negative $M$ for spin-up and spin-down electrons, respectively. From the above discussions, the spin-up (down) electrons show normal oscillating (bending) trajectory (see Figs. 3). Therefore, the edge states only appear for spin-down electrons, i.e., the quantum anomalous spin Hall effect. Similar behavior can also be found in three-dimensional topological insulators, because the form of the Hamiltonian of 3D TI is almost the same as that of $2 \mathrm{D}$ TI. Therefore one can expect that the bending trajectory of electrons in 3D TIs will lead to the topological surface states.

In a spin-orbit system, usually electron spin can be ma-

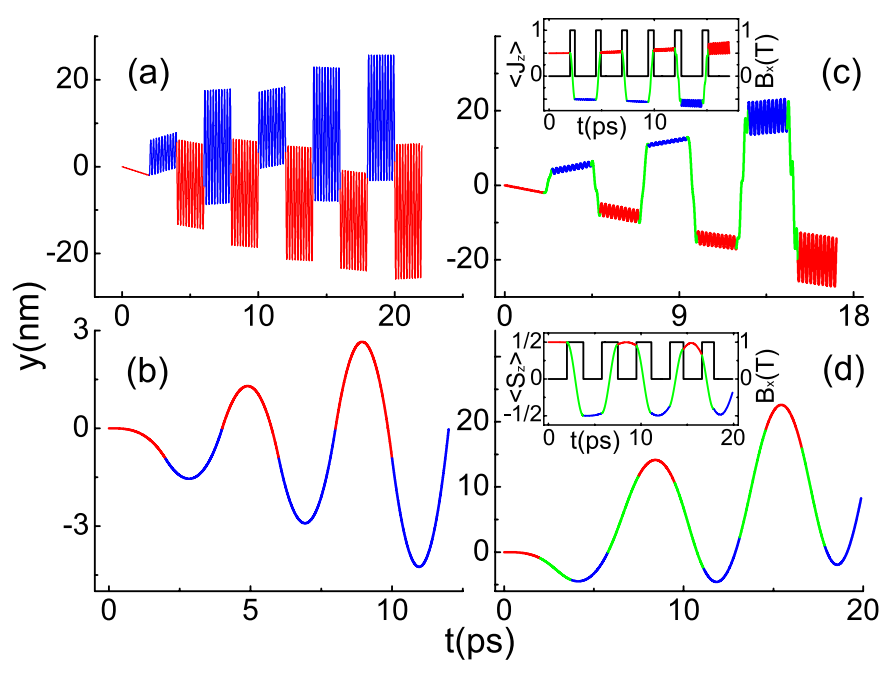

FIG. 4: (color online). The trajectories of a initial spin-up electron driven by a uniform electric field in $\mathrm{HgTe}$ QW (a) and a GaAs 2DEG with the SOIs (b). The red and blue curves denote the spin-up and spin-down electrons, respectively. The electron spin is flipped periodically at a time interval $t=2 \mathrm{ps}$. (c) and (d), the same as (a) and (b), but for a realistic case: a series of the square- shaped magnetic field pulses with the magnitude $B_{0}=1 \mathrm{~T}$. The parameters are the same with those in Fig. $3, k_{0}=0$.

nipulated by control its orbital motion, such as spin transistor [27]. And vise versa, it is also possible to control electron orbital motion by manipulating electron spin. In a 2D TI, e.g., a 2DEG in a HgTe QW with an inverted band structure, electron spin flipping leads to an interesting quantum side jump (see Fig. 4(a)), which is the manifestation of the spin-dependent $\mathrm{ZB}$ and caused by the spin-dependent driving force. While in the $2 \mathrm{DEG}$ with the SOIs, an interesting snake orbit motion can be found (see Fig. 4(b)). This ultrafast spin flipping process can be achieved by applying a series of specifically shaped magnetic field pulses (see the insets in Figs. 4(c) and $4(\mathrm{~d})$ ), which was used to flip the magnetization of the magnetic random access memory devices [28]. The width of the magnetic feild pulses is determined by the magnitudes of the magnetic field pulses $B_{0}$, usually it will take longer (shorter) time to flip electron spins for weak (strong) magnetic field $B_{0}$.

Finally, we comment on how to detect these interesting orbital motions experimentally. We propose a nearfield scanning optical microscope (NSOM) technique in microwave or $\mathrm{THz}$ regime to detect the edge states, $\mathrm{ZB}$, quantum side jump and snake-orbit motions. The NSOM technique breaks the far field resolution limit by exploiting the properties of evanescent waves. With this technique, the resolution of the image is limited by the size of the detector aperture and not by the wavelength of the illuminating light. A spatial lateral resolution of the NSOM can approach to $20 \mathrm{~nm}$ [29]. A microwave light beam is applied at a specific spatial position through a 
NSOM (see Fig. 1(b)), the absorption of the microwave beam changes when electrons pass below. This experimental technique was used to detect the topological edge states in a HgTe QW very recently.

In summary, we give an intuitive physical picture about the dynamical origin of the edge states in TIs, a skipping orbit motion. By flipping electron spin quickly, one can control the electron orbital motion efficiently. An interesting quantum side jump and snake orbital motion can be found in the TIs and 2DEGs with the SOIs. This feature provides us a new way to control electron orbital motion by manipulating electron spin.

This work was supported by the NSFC Grants No. 10934007 and the grant No. 2011CB922204 from the MOST of China, and S.C.Z. is supported by the DARPA Program on Topological Insulators.

[1] X. L. Qi and S. C. Zhang, Phys. Today 63, 33 (2010).

[2] M. Z. Hasan and C. L. Kane, Rev. Mod. Phys. 82, 3045 (2010).

[3] C. L. Kane, E. J. Mele, Phys. Rev. Lett. 95, 146802 (2005).

[4] B. A. Bernevig, T. L. Hughes, S. C. Zhang, Science 314, 1757 (2006).

[5] M. König, S. Wiedmann, C. Brüne, A. Roth, H. Buhmann, L. W. Molenkamp, X. L. Qi, S. C. Zhang, Science 318, 766 (2007).

[6] D. Hsieh, D. Qian, L. Wray, Y. Xia, Y. S. Hor, R. J. Cava, M. Z. Hasan, Nature (London) 452, 970 (2008).

[7] Y. L. Chen, J. G. Analytis, J. H. Chu, Z. K. Liu, S. K. Mo, X. L. Qi, H. J. Zhang, D. H. Lu, X. Dai, Z. Fang, S. C. Zhang, I. R. Fisher, Z. Hussain, Z. X. Shen, Science 352, 178 (2009).

[8] C. X. Liu, X. L. Qi, Xi Dai, Z. Fang and S. C. Zhang, Phys. Rev. Lett. 101, 146802 (2008).

[9] J. Schliemann, D. Loss, and R. M. Westervelt, Phys. Rev. Lett. 94, 206801 (2005).

[10] T. M. Rusin, W. Zawadzki, Phys. Rev. B 76, 195439 (2007).

[11] Y. Xia, D. Qian, D. Hsieh, L. Wray, A. Pal, H. Lin, A. Bansil, D. Grauer, Y. S. Hor, R. J. Cava and M. Z. Hasan, Nature Phys. 5, 438 (2009).
[12] H. J. Zhang, C. X. Liu, X. L. Qi, X. Dai, Z. Fang and S. C. Zhang, Nature Phys. 5, 438 (2009).

[13] E. Shrödinger, Sitzungsb. Preuss. Akad. Wiss. Phys.Math. K1. 24, 418 (1930).

[14] K. Huang, Am. J. Phys. 20, 479 (1952).

[15] J. Schliemann, Phys. Rev. B. 75, 045304 (2007).

[16] F. Cannata, L. Ferrari, and G. Russo, Solid State Commun. 74, 309 (1990); L. Ferrari and G. Russo, Phys. Rev. B 42, 7454 (1990); F. Cannata and L. Ferrari, Phys. Rev. B 44, 8599 (1991).

[17] D. Lurié and S. Cremer, Physica (Amsterdam) 50, 224 (1970).

[18] R. Winkler, U. Zülicke and J. Bolte, Phys. Rev. B. 75, 205314 (2007); J. Cserti, G. Dávid, Phys. Rev. B. 82, 201405 (2010); W. Zawadzki, T. M. Rusin, J. Phys. Condens. Matter 23, 143201 (2011).

[19] L. Lamata, J. León, T. Schätz, and E. Solano, Phys. Rev. Lett. 98, 253005 (2007).

[20] A. Bermudez, M. A. Martin-Delgado, and E. Solano, Phys. Rev. A. 76, 041801(R) (2007).

[21] R. Gerritsma, G. Kirchmair, F. Zähringer, E. Solano, R. Blatt, C. F. Roos, Nature (London) 463, 68 (2010).

[22] J. Y. Vaishnav, C. W. Clark, Phys. Rev. Lett. 100, 153002 (2008)

[23] M. König, H. Buhmann, L. W. Molenkamp, T. Hughes, C. X. Liu, X. L. Qi, S. C. Zhang, J. Phys. Soc. Jpn. 77, 031007 (2008).

[24] S. Murakami, N. Nagaosa, S. C. Zhang, Science 301, 1348 (2003).

[25] Z. F. Jiang, R. D. Li, S. C. Zhang, and W. M. Liu, Phys. Rev. B. 72, 045201 (2005).

[26] L. Fu and C. L. Kane, Phys. Rev. Lett. 100, 096407 (2008); B. Zhou, H. Z. Lu, R. L. Chu, S. Q. Shen, and Q. Niu, Phys. Rev. Lett. 101, 246807 (2008); E. G. Novik, P. Recher, E. M. Hankiewicz, and B. Trauzettel, Phys. Rev. B. 81, 241303 (2010) K. Chang and W. K. Lou, Phys. Rev. Lett. 106, 206802 (2011).

[27] S. Datta and B. Das, Appl. Phys. Lett. 56, 665 (1990); H. C. Koo, J. H. Kwon, J. Eom, J. Chang, S. H. Han, and M. Johnson, Science 325, 1515 (2009).

[28] Th. Gerrits, H. A. M. van den Berg, J. Hohlfeld, L. Bär, and Th. Rasing, Nature (London), 418, 509 (2002); M. R. Freeman, R. R. Ruf, and R. J. Gambino, IEEE TRANS. MAG. 27, 4840 (1991).

[29] Y. Oshikane T. Kataoka, M. Okuda, S. Hara, H. Inoue, M. Nakano, Sci. Technol. Adv. Mater. 8, 181 (2007). 


\title{
Online Supplemental Material: Anomalous Electron Trajectory in Topological Insulators
}

\author{
Likun $\mathrm{Shi}^{1}$, Shoucheng Zhang ${ }^{2}$, Kai Chang ${ }^{11,2}$ \\ ${ }^{11}$ SKLSM, Institute of Semiconductors, Chinese Academy of Sciences, P.O. Box 912, Beijing 100083, China \\ ${ }^{22}$ Department of Physics, Stanford University, Stanford, CA94305
}

\section{DIAGRAM TECHNIQUE IN THE ABSENCE OF EXTERNAL FIELD}

We consider a single-particle Hamiltonian of electron in a general form

$$
\begin{aligned}
H & =\epsilon(k)+\sum_{i} d_{i}(k) \sigma^{i} \\
& =\epsilon(k)+D(k)
\end{aligned}
$$

where $\epsilon(k)$ denotes the kinetic energy and the second term describes the spin-orbit interactions or interband coupling.

In the Heisenberg picture, the position operator of electron $y_{H}(t)$ evolving with time $t$ can be obtained by

$$
\begin{aligned}
y_{H}(t) & =e^{i H t / \hbar} y e^{-i H t / \hbar}, \\
& =y(0)+(i t / \hbar)[H, y]+\frac{(i t / \hbar)^{2}}{2 !}[H,[H, y]]+\cdots, \\
& =y(0)+(i t / \hbar) \epsilon^{y}+\sum_{n=1} \frac{(i t / \hbar)^{n}}{n !} T_{n},
\end{aligned}
$$

where we show the notions explicitly:

$$
\begin{gathered}
\epsilon^{y}=[\epsilon, y]=-i \partial_{k_{y}} \epsilon, \\
T_{1}=[H, y]=[D, y], \\
T_{2}=\left[H, T_{1}\right]=\left[D, T_{1}\right], \\
\vdots \\
T_{n}=\left[D, T_{n-1}\right] .
\end{gathered}
$$

The above equation involves the infinite summation of the commutators $T_{n}$ and therefore it is not easy to obtain the analytical expression of the position operator $y_{H}(t)$. In order to calculate the commutation in arbitrary order and the summation, we develop a diagram technique as follow which makes the lengthy calculation of the commutation much easier. Utilizing the similarity between the commutation relationship $\left[\sigma_{i}, \sigma_{j}\right]=2 i \varepsilon_{i j k} \sigma_{k}$ and the vector cross production $\boldsymbol{A} \times \boldsymbol{B}=\varepsilon_{i j k} A_{i} B_{j} \boldsymbol{e}_{k}$, we obtain the following relationship

$$
\begin{aligned}
D(k)=d_{1} \sigma^{1}+d_{2} \sigma^{2}+d_{3} \sigma^{3} & \rightarrow \boldsymbol{D}=\left(d_{1}, d_{2}, d_{3}\right), \\
T_{1}=[H, y]=t_{0}+t_{1} \sigma^{1}+t_{2} \sigma^{2}+t_{3} \sigma^{3} & \rightarrow \boldsymbol{T}_{1}=\left(t_{1}, t_{2}, t_{3}\right), \\
T_{2}=\left[H, T_{1}\right]=\left[D, T_{1}\right] & \rightarrow 2 i\left(\boldsymbol{D} \times \boldsymbol{T}_{1}\right), \\
& \cdots \\
T_{n+1}=\left[H, T_{n}\right]=\left[D, T_{n}\right] & \rightarrow 2 i\left(\boldsymbol{D} \times \boldsymbol{T}_{n}\right) .
\end{aligned}
$$

These relationships can be illustrated in Fig. 1. From this figure, one can see that the vectors $\boldsymbol{T}_{2 n+1}$ and $\boldsymbol{T}_{2 n}$ $(n=1,2,3 \cdots)$ point along the two perpendicular axes, respectively, but the magnitudes (or lengths) of the vectors $\boldsymbol{T}_{2 n+1}$ and $\boldsymbol{T}_{2 n}$ are both geometrical series. This character allows us to get the analytical expression of the infinite summation. 


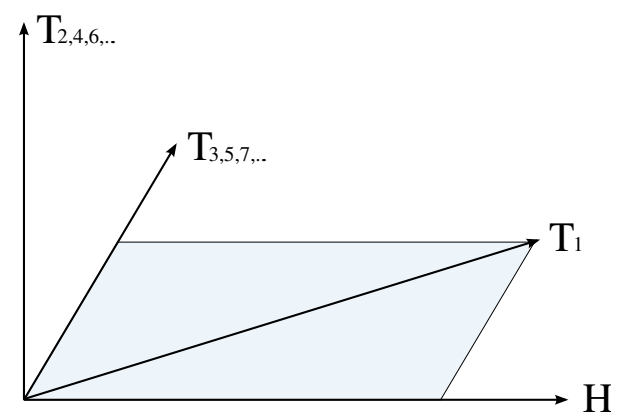

FIG. 1: Illustrating figure showing how to calculate $T_{n}$ using the vector cross times (see Eq.[4]).

Summing up all the terms, we get an analytical expression for the position operator of electron

$$
\begin{aligned}
y_{H}(t) & =y(0)+\frac{i t}{\hbar}\left(\epsilon^{y}+T_{1}-\frac{T_{3}}{|D|^{2}}\right) \\
& +\frac{T_{2}}{2|D|^{2}}[\cos (2|D| t / \hbar)-1] \\
& +\frac{i T_{3}}{2|D|^{3}}[\sin (2|D| t / \hbar)],
\end{aligned}
$$

where $|D|=\sqrt{d_{1}^{2}+d_{2}^{2}+d_{3}^{2}}$.

\section{ANALYTICAL EXPRESSION FOR ELECTRON TRAJECTORY: ADIABATIC APPROXIMATION}

The Hamiltonian for a two-dimensional topological insulator, a HgTe quantum well with an inverted band structure described by the BHZ model, under a uniform electric filed is

$$
H(k)_{\uparrow \downarrow}=H_{0}(k)_{\uparrow \downarrow}+V\left(x_{i}\right),
$$

where $H_{0}(k)_{\uparrow \downarrow}=C-D k^{2} \pm A k_{x} \sigma^{x}+A k_{y} \sigma^{y}+\left(M-B k^{2}\right) \sigma^{z}, V(\mathbf{x})=-e \mathbf{E} \cdot \mathbf{r} \sigma^{z}$. After the proper unitary transformation $U(k)_{\uparrow \downarrow}$ which can diagonalize the $H_{0}(k)$, i. e., $\tilde{H}_{0}=U(k)_{\uparrow \downarrow} H_{0}(k)_{\uparrow \downarrow} U^{\dagger}(k)_{\uparrow \downarrow}$, then $H_{\uparrow \downarrow}^{\text {eff }}=\tilde{H}_{0}(k)-e \sum_{i=x, y} E_{i} D_{i, \uparrow \downarrow}$, where

$$
\begin{aligned}
& \tilde{H}_{0}(k)=C-D k^{2}+\left(\begin{array}{cc}
-\sqrt{A^{2} k^{2}+\left(M-B k^{2}\right)^{2}} & 0 \\
0 & \sqrt{A^{2} k^{2}+\left(M-B k^{2}\right)^{2}}
\end{array}\right) \\
& U(k)_{\uparrow \downarrow}=\left(\begin{array}{ccc}
\frac{\mp\left[-B k^{2}+M-\sqrt{A^{2} k^{2}+\left(M-B k^{2}\right)^{2}}\right]}{A\left(k_{x} \pm i k_{y}\right) \sqrt{1+\frac{\left(B k^{2}-M+\sqrt{A^{2} k^{2}+\left(M-B k^{2}\right)^{2}}\right)}{A^{2} k^{2}}}} & \frac{-B k^{2}+M+\sqrt{A^{2} k^{2}+\left(M-B k^{2}\right)^{2}}}{A\left(k_{x} \pm i k_{y}\right) \sqrt{1+\frac{\left(B k^{2}+M+\sqrt{A^{2} k^{2}+\left(M-B k^{2}\right)^{2}}\right)}{A^{2} k^{2}}}} \\
\frac{1}{\sqrt{1+\frac{\left(B k^{2}-M+\sqrt{A^{2} k^{2}+\left(M-B k^{2}\right)^{2}}\right)}{A^{2} k^{2}}}} & \frac{1}{\sqrt{1+\frac{\left(-B k^{2}+M+\sqrt{A^{2} k^{2}+\left(M-B k^{2}\right)^{2}}\right)}{A^{2} k^{2}}}}
\end{array}\right),
\end{aligned}
$$

and

$$
\tilde{A}_{i}(k)_{\uparrow \downarrow}=-i \cdot U(k)_{\uparrow \downarrow} \partial_{k_{i}} U^{\dagger}(k)_{\uparrow \downarrow} \cdot U(k)_{\uparrow \downarrow} \sigma^{z} U^{\dagger}(k)_{\uparrow \downarrow} \cdot
$$

The analytical expression is too lengthy to be omitted here. Adopting the adiabatic approximation, we neglect the interband transitions, i.e., neglecting the off-diagonal matrix elements of $\tilde{A}$,

$$
F_{x y}(k)_{\uparrow \downarrow}=i\left[D_{i}, D_{j}\right]=\left(\begin{array}{cc} 
\pm \frac{A^{2}\left(M^{2}-B^{2} k^{4}\right)}{2\left[A^{2} k^{2}+\left(M-B k^{2}\right)^{2}\right]^{2}} & 0 \\
0 & \pm \frac{A^{2}\left(M^{2}-B^{2} k^{4}\right)}{2\left[A^{2} k^{2}+\left(M-B k^{2}\right)^{2}\right]^{2}}
\end{array}\right) .
$$


The equation of motion for the spin-up/down $(\uparrow \downarrow)$ negative/positive branch $(\lambda= \pm 1)$ can be written as:

$$
\begin{gathered}
k_{i}=k_{i 0}+\lambda \frac{e E_{i}}{\hbar} t \\
\frac{d x_{\uparrow \downarrow}}{d t}=-\frac{2 D}{\hbar} k_{x}+\lambda \frac{2 B\left(M-B k^{2}\right)-A^{2}}{\hbar \sqrt{A^{2} k^{2}+\left(M-B k^{2}\right)^{2}}} k_{x} \pm \frac{e E_{y}}{\hbar} \frac{A^{2}\left(M^{2}-B^{2} k^{4}\right)}{2\left[A^{2} k^{2}+\left(M-B k^{2}\right)^{2}\right]^{2}}, \\
\frac{d y_{\uparrow \downarrow}}{d t}=-\frac{2 D}{\hbar} k_{y}+\lambda \frac{2 B\left(M-B k^{2}\right)-A^{2}}{\hbar \sqrt{A^{2} k^{2}+\left(M-B k^{2}\right)^{2}}} k_{y} \mp \frac{e E_{x}}{\hbar} \frac{A^{2}\left(M^{2}-B^{2} k^{4}\right)}{2\left[A^{2} k^{2}+\left(M-B k^{2}\right)^{2}\right]^{2}} .
\end{gathered}
$$

When $E_{y}=k_{y}=0$, the integration of the topological term [1], $\mp \frac{e E_{x}}{\hbar} \frac{A^{2}\left(M^{2}-B^{2} k^{4}\right)}{2\left[A^{2} k^{2}+\left(M-B k^{2}\right)^{2}\right]^{2}}$, represents the electron's orbital motion in the $y$ axis brought by the effective field strength $F_{x y}$,

$$
y_{\uparrow \downarrow}^{\text {orb }}=\int d k \frac{A^{2}\left(M^{2}-B^{2} k^{4}\right)}{2\left[A^{2} k^{2}+\left(M-B k^{2}\right)^{2}\right]^{2}} .
$$

To the second order of $k$,

$$
y_{\uparrow \downarrow}^{\text {orb }}=\mp \frac{A^{2}}{2 M^{2}} k+O(k)^{3}
$$

and for the $k \rightarrow \infty$ limit,

$$
y_{\uparrow \downarrow}^{\mathrm{orb}} \rightarrow \mp \frac{i A \pi\left(\sqrt{\frac{B^{2}}{A^{2}-2 B M-i A \sqrt{-A^{2}+4 B M}}}-\sqrt{\frac{B^{2}}{A^{2}-2 B M+i A \sqrt{-A^{2}+4 B M}}}\right)}{4 \sqrt{-2 A^{2}+8 B M}},
$$

which is about $14.31 \mathrm{~nm}$ adopting the parameters in Ref. [2]. We can see that this topological shift have a upper limit during a single ballistic motion.

Next, we derive the analytical expression for the trembling motion, i.e., the Zitterbewegung [3]. We assume the wave function has the form of $|\psi(x, t)\rangle=\exp \left(-i e \mathbf{E} \cdot \mathbf{r} \sigma_{z} t / \hbar\right)|u(x, t)\rangle$, and substitute $|\psi(x, t)\rangle$ into the Schrödinger equation

$$
i \hbar \partial_{t}|\psi(x, t)\rangle=\left[H_{0}(k)_{\uparrow \downarrow}+e \mathbf{E} \cdot \mathbf{r} \sigma_{z}\right]|\psi(x, t)\rangle
$$

where $H_{0}(k)_{\uparrow \downarrow}=C-D k^{2} \pm A k_{x} \sigma^{x}+A k_{y} \sigma^{y}+\left(M-B k^{2}\right) \sigma^{z}$, because

$$
\begin{aligned}
i \hbar \partial_{t}|\psi(x, t)\rangle & =\left[e \mathbf{E} \cdot \mathbf{r} \sigma_{z}\right]|\psi(x, t)\rangle \\
+ & \exp \left(-i e \mathbf{E} \cdot \mathbf{r} \sigma_{z} t / \hbar\right)\left[i \hbar \partial_{t}|u(x, t)\rangle\right],
\end{aligned}
$$

we get a time-dependent Schrödinger equation

$$
i \hbar \partial_{t}|u(x, t)\rangle=H_{0}(k, t)|u(x, t)\rangle
$$

where $k_{i}(t)=k_{i 0}+\lambda \frac{e E_{i}}{\hbar} t$. We assume that $|u(x, t)\rangle=\sum_{\lambda} C_{\lambda}(t) \exp \left(-i \alpha_{\lambda}\right) U^{\dagger}(k)|\lambda\rangle$, where $|\lambda\rangle$ represents any eigenstate of $S_{z}$, that is $S_{z}|\lambda\rangle=\lambda|\lambda\rangle$, so $U^{\dagger}(k)_{\uparrow \downarrow}|\lambda\rangle$ is the instant eigenstate of $H_{0}(k)_{\uparrow \downarrow}, H_{0}(k)_{\uparrow \downarrow} U^{\dagger}(k)_{\uparrow \downarrow}|\lambda\rangle=$ $\epsilon_{\lambda}(t) U^{\dagger}(k)_{\uparrow \downarrow}|\lambda\rangle$, where $\epsilon_{\frac{1}{2} / \frac{3}{2}}(t)=C-D k^{2} \mp \sqrt{A^{2} k^{2}+\left(M-B k^{2}\right)^{2}} \cdot \alpha_{\lambda}=(1 / \hbar) \int_{0}^{t} \epsilon_{\lambda}\left(t^{\prime}\right) d t^{\prime}$. Because

$$
\begin{aligned}
i \hbar \partial_{t}|u(x, t)\rangle & =i \hbar \sum_{\lambda}\left[\partial_{t} C_{\lambda}(t)\right] \exp \left(-i \alpha_{\lambda}\right) U^{\dagger}(k)_{\uparrow \downarrow}|\lambda\rangle \\
& +\sum_{\lambda} C_{\lambda}(t)\left[\epsilon_{\lambda}(t) \exp \left(-i \alpha_{\lambda}\right)\right] U^{\dagger}(k)_{\uparrow \downarrow}|\lambda\rangle \\
& +i \hbar \sum_{\lambda} C_{\lambda}(t) \exp \left(-i \alpha_{\lambda}\right)\left[\partial_{t} U^{\dagger}(k)_{\uparrow \downarrow}\right]|\lambda\rangle,
\end{aligned}
$$

and

$$
H_{0}|u(x, t)\rangle=\sum_{\lambda} C_{\lambda}(t)\left[\epsilon_{\lambda}(t) \exp \left(-i \alpha_{\lambda}\right)\right] U^{\dagger}(k)_{\uparrow \downarrow}|\lambda\rangle
$$


thus

$$
\sum_{\lambda}\left[\partial_{t} C_{\lambda}(t)\right] \exp \left(-i \alpha_{\lambda}\right) U^{\dagger}(k)_{\uparrow \downarrow}|\lambda\rangle+\sum_{\lambda} C_{\lambda}(t) \exp \left(-i \alpha_{\lambda}\right)\left[\partial_{t} U^{\dagger}(k)_{\uparrow \downarrow}\right]|\lambda\rangle=0 .
$$

Act $U(k)_{\uparrow \downarrow}$ and $\left\langle\lambda^{\prime}\right|$ to the left side,

$$
\left[\partial_{t} C_{\lambda^{\prime}}(t)\right] \exp \left(-i \alpha_{\lambda^{\prime}}\right)+\sum_{\lambda} C_{\lambda}(t) \exp \left(-i \alpha_{\lambda}\right)\left\langle\lambda^{\prime}\left|U(k)_{\uparrow \downarrow} \partial_{t} U^{\dagger}(k)_{\uparrow \downarrow}\right| \lambda\right\rangle=0 .
$$

For the helicity state, $|1 / 2\rangle=(1,0)^{T},|3 / 2\rangle=(0,1)^{T}$, we set the initial state $C_{1 / 2}(0)=1, C_{3 / 2}(0)=0$. The adiabatic approximation assumes that $0 \simeq C_{3 / 2}(t) \ll C_{1 / 2}(t)$ is always satisfied, which leads to

$$
\begin{aligned}
\partial_{t} C_{1 / 2}(t) & \simeq-C_{1 / 2}(t)\left\langle 1 / 2\left|U(k)_{\uparrow \downarrow} \partial_{t} U^{\dagger}(k)_{\uparrow \downarrow}\right| 1 / 2\right\rangle, \\
\partial_{t} C_{3 / 2}(t) & \simeq-C_{1 / 2}(t) \exp (i \Delta \alpha)\left\langle 3 / 2\left|U(k)_{\uparrow \downarrow} \partial_{t} U^{\dagger}(k)_{\uparrow \downarrow}\right| 1 / 2\right\rangle, \\
\Delta \alpha & =\alpha_{3 / 2}-\alpha_{1 / 2}=(1 / \hbar) \int_{0}^{t} \Delta \epsilon\left(t^{\prime}\right) d t^{\prime} .
\end{aligned}
$$

Because $y_{\uparrow \downarrow}^{\mathrm{ZB}}=C_{3 / 2}^{*} C_{1 / 2} e^{-i \Delta \alpha}\left\langle 3 / 2\left|i U(k)_{\uparrow \downarrow} \partial_{k_{y}} U^{\dagger}(k)_{\uparrow \downarrow}\right| 1 / 2\right\rangle+$ h.c., Then we can calculate the $U(k)_{\uparrow \downarrow} \partial_{t} U^{\dagger}(k)_{\uparrow \downarrow}$ and $U(k)_{\uparrow \downarrow} \partial_{k_{y}} U^{\dagger}(k)_{\uparrow \downarrow}$. When $E_{y}=\partial k_{y} / \partial t=0$,

$$
U(k)_{\uparrow \downarrow} \partial_{t} U^{\dagger}(k)_{\uparrow \downarrow}=U(k)_{\uparrow \downarrow} \frac{\partial}{\partial k_{x}} U^{\dagger}(k)_{\uparrow \downarrow} \cdot \frac{\partial k_{x}}{\partial t},
$$

at small $k$,

$$
\begin{aligned}
& \left.U(k)_{\uparrow \downarrow} \frac{\partial}{\partial k_{x}} U^{\dagger}(k)_{\uparrow \downarrow}\right|_{k=0}=\left[\begin{array}{cc}
0 & \pm \frac{A}{2 M} \\
\mp \frac{A}{2 M} & 0
\end{array}\right]+O\left(k^{2}\right), \\
& \left.U(k)_{\uparrow \downarrow} \frac{\partial}{\partial k_{y}} U^{\dagger}(k)_{\uparrow \downarrow}\right|_{k=0}=\left[\begin{array}{cc}
0 & -\frac{i A}{2 M} \\
-\frac{i A}{2 M} & 0
\end{array}\right]+O\left(k^{2}\right),
\end{aligned}
$$

Notice that the $U(k)_{\uparrow \downarrow} \partial_{k_{y}} U^{\dagger}(k)_{\uparrow \downarrow}$ and $\left\langle 3 / 2\left|i U(k)_{\uparrow \downarrow} \partial_{k_{y}} U^{\dagger}(k)_{\uparrow \downarrow}\right| 1 / 2\right\rangle$ is only finite for the diagonal and off-diagonal matrix elements, respectively. Therefore

$$
\begin{aligned}
\partial_{t} C_{1 / 2}(t)_{\uparrow \downarrow} & =0, \\
\partial_{t} C_{3 / 2}(t)_{\uparrow \downarrow} & =\mp \frac{A}{2 M} \frac{e E_{x}}{\hbar} \exp (i \Delta \alpha) .
\end{aligned}
$$

Adopting the adiabatic approximation, $\epsilon_{\lambda}(t)$ are slowly varying functions of the time $t$,

$$
C_{3 / 2}(t)_{\uparrow \downarrow}=\mp \frac{A}{2 M} \frac{e E_{x}}{\Delta \epsilon(t)} \exp (i \Delta \alpha) .
$$

Finally we get

$$
y_{\uparrow \downarrow}^{\mathrm{ZB}}= \pm \frac{A^{2}}{2 M^{2}} \frac{e E_{x}}{\Delta \epsilon(t)} \sin \left(\frac{\Delta \epsilon(t)}{\hbar} t\right),
$$

where $\Delta \epsilon(t)=2 \sqrt{A^{2} k^{2}+\left(M-B k^{2}\right)^{2}}=2|M|+O\left(k^{2}\right)$. At small $k$,

$$
y_{\uparrow \downarrow}^{\mathrm{ZB}} \simeq \pm \frac{A^{2}}{4 M^{2}} \frac{e E_{x}}{|M|} \sin \left(\frac{\Delta \epsilon(t)}{\hbar} t\right) .
$$

When we consider the trajectory of an initially resting electron/hole in 2D TI under an AC electric field in the $x$-direction $E_{x}=E_{0} \cos (\omega t / \hbar)$, the equation of orbital motion for the spin-up/down ( $\left.\downarrow\right)$ electron/hole $(\lambda= \pm 1)$ is

$$
\begin{gathered}
k_{x}=\lambda \frac{e E_{0}}{\hbar} \frac{\hbar}{\omega} \sin (\omega t / \hbar), \\
\frac{d x_{\uparrow \downarrow}}{d t}=-\frac{2 D}{\hbar} k_{x}+\lambda \frac{2 B\left(M-B k^{2}\right)-A^{2}}{\hbar \sqrt{A^{2} k^{2}+\left(M-B k^{2}\right)^{2}}} k_{x}, \\
\frac{d y_{\uparrow \downarrow}}{d t}=\mp \frac{e E_{x}}{\hbar} \frac{A^{2}\left(M^{2}-B^{2} k^{4}\right)}{2\left[A^{2} k^{2}+\left(M-B k^{2}\right)^{2}\right]^{2}} .
\end{gathered}
$$


To the second order of $k$,

$$
\begin{aligned}
\frac{d x_{\uparrow \downarrow}}{d t} & =-\frac{2 D}{\hbar} k_{x}+\lambda \frac{2 B M-A^{2}}{\hbar|M|} k_{x}, \\
\frac{d y_{\uparrow \downarrow}}{d t} & =\mp \frac{e E_{x}}{\hbar} \frac{A^{2}}{2 M^{2}},
\end{aligned}
$$

then we can get the orbital motion under the adiabatic approximation

$$
\begin{aligned}
& x_{\uparrow \downarrow}^{\text {orb }}=\frac{e E_{0}}{\hbar^{2}}\left(\lambda \frac{A^{2}-2 B M}{|M|}+2 D\right)\left(\frac{\hbar}{\omega}\right)^{2}[\cos (\omega t / \hbar)-1], \\
& y_{\uparrow \downarrow}^{\text {orb }}=\mp \frac{A^{2}}{2 M^{2}} \frac{e E_{0}}{\hbar} \frac{\hbar}{\omega} \sin (\omega t / \hbar) .
\end{aligned}
$$

\section{THE NUMERICAL CALCULATION OF ELECTRON TRAJECTORY $y(t)$}

We assume that the electric field $E=(-E, 0,0)$ points along the $-x$ axis without loss of any generality, thus

$$
H_{\uparrow \downarrow}(k)=C-D k^{2}+\left( \pm A k_{x}, A k_{y}, M-B k^{2}\right) \cdot \sigma-e E x \cdot \sigma_{z},
$$

or

$$
H(k)=C-D k^{2}+A k_{x} \cdot s_{1}+A k_{y} \cdot s_{2}+\left(M-B k^{2}\right) \cdot s_{3}-e E x \cdot s_{3},
$$

where we define

$$
\begin{gathered}
s_{1}=\left[\begin{array}{cc}
\sigma_{x} & 0 \\
0 & -\sigma_{x}
\end{array}\right], s_{2}=\left[\begin{array}{cc}
\sigma_{y} & 0 \\
0 & \sigma_{y}
\end{array}\right], s_{3}=\left[\begin{array}{cc}
\sigma_{z} & 0 \\
0 & \sigma_{z}
\end{array}\right], \\
s_{4}=\left[\begin{array}{cc}
\sigma_{x} & 0 \\
0 & \sigma_{x}
\end{array}\right], s_{5}=\left[\begin{array}{cc}
\sigma_{y} & 0 \\
0 & -\sigma_{y}
\end{array}\right], s_{6}=\left[\begin{array}{cc}
\sigma_{z} & 0 \\
0 & -\sigma_{z}
\end{array}\right],
\end{gathered}
$$

then

$$
\begin{gathered}
\frac{d\left\langle k_{x}\right\rangle}{d t}=\frac{e E}{\hbar}\left\langle s_{3}\right\rangle, \\
\frac{d\left\langle k_{y}\right\rangle}{d t}=0, \\
\frac{d\langle x\rangle}{d t}=-\frac{2 D}{\hbar}\left\langle k_{x}\right\rangle+\frac{A}{\hbar}\left\langle s_{1}\right\rangle-\frac{2 B}{\hbar}\left\langle k_{x}\right\rangle\left\langle s_{3}\right\rangle, \\
\frac{d\langle y\rangle}{d t}=-\frac{2 D}{\hbar}\left\langle k_{y}\right\rangle+\frac{A}{\hbar}\left\langle s_{2}\right\rangle-\frac{2 B}{\hbar}\left\langle k_{y}\right\rangle\left\langle s_{3}\right\rangle, \\
\frac{d\left\langle s_{1}\right\rangle}{d t}=-2 \frac{M-B\langle k\rangle^{2}-e E\langle x\rangle}{\hbar}\left\langle s_{5}\right\rangle+\frac{2 A}{\hbar}\left\langle k_{y}\right\rangle\left\langle s_{6}\right\rangle, \\
\frac{d\left\langle s_{2}\right\rangle}{d t}=2 \frac{M-B\langle k\rangle^{2}-e E\langle x\rangle}{\hbar}\left\langle s_{4}\right\rangle-\frac{2 A}{\hbar}\left\langle k_{x}\right\rangle\left\langle s_{6}\right\rangle, \\
\frac{d\left\langle s_{3}\right\rangle}{d t}=-\frac{2 A}{\hbar}\left\langle k_{y}\right\rangle\left\langle s_{4}\right\rangle+\frac{2 A}{\hbar}\left\langle k_{x}\right\rangle\left\langle s_{5}\right\rangle, \\
\frac{d\left\langle s_{4}\right\rangle}{d t}=-2 \frac{M-B\langle k\rangle^{2}-e E\langle x\rangle}{\hbar}\left\langle s_{2}\right\rangle+\frac{2 A}{\hbar}\left\langle k_{y}\right\rangle\left\langle s_{3}\right\rangle, \\
\frac{d\left\langle s_{5}\right\rangle}{d t}=2 \frac{M-B\langle k\rangle^{2}-e E\langle x\rangle}{\hbar}\left\langle s_{1}\right\rangle-\frac{2 A}{\hbar}\left\langle k_{x}\right\rangle\left\langle s_{3}\right\rangle, \\
\frac{d\left\langle s_{6}\right\rangle}{d t}=-\frac{2 A}{\hbar}\left\langle k_{y}\right\rangle\left\langle s_{1}\right\rangle+\frac{2 A}{\hbar}\left\langle k_{x}\right\rangle\left\langle s_{2}\right\rangle .
\end{gathered}
$$


For a given initial state, we can calculate the electron trajectory $\langle y(t)\rangle$ involving with the time $t$ numerically.

[1] Murakami, S., Nagaosa, N., Zhang, S-C. Science 301, 1348 (2003).

[2] König, M., Buhmann, H., Molenkamp, L. W., Hughes, T. L., Liu, C. X., Qi, X. L., Zhang, S-C. J. Phys. Soc. Jpn. 77, 031007 (2008)

[3] Jiang, Z. F., Li, R. D., Zhang, S-C., and Liu, W. M. Phys. Rev. B. 72, 045201 (2005). 